\title{
Self-harm in postpartum depression and referrals to a perinatal mental health team: an audit study
}

\author{
Christine Healey • Richard Morriss • Carol Henshaw • \\ Ovais Wadoo • Aamer Sajjad • Helen Scholefield • \\ Peter Kinderman
}

Received: 2 October 2012 / Accepted: 13 February 2013 /Published online: 6 March 2013

(C) The Author(s) 2013. This article is published with open access at Springerlink.com

\begin{abstract}
There is a paucity of research on self-harm during pregnancy and the postpartum period despite suicide being a leading cause of death and high rates of mental disorder during this time. This audit describes a cohort of women referred to a new perinatal mental health team (PMHT) based in a large maternity hospital in the UK over a 12month period. The audit was conducted in two stages. Stage one describes the clinical and socio-demographic characteristics of 225 pregnant women referred to the team after screening positive for a significant mental health history.
\end{abstract}

C. Healey $(\square) \cdot$ P. Kinderman

University of Liverpool, Waterhouse Building,

Block B, 2nd Floor,

Liverpool, UK

e-mail: chealey@liverpool.ac.uk

P. Kinderman

e-mail: p.kinderman@liverpool.ac.uk

R. Morriss

University of Nottingham, Nottingham, UK

e-mail: Richard.Morriss@nottingham.ac.uk

C. Henshaw $\cdot$ H. Scholefield

Liverpool Women's Hospital, Liverpool, UK

C. Henshaw

e-mail: chenshaw@doctors.org.uk

H. Scholefield

e-mail: Helen.Scholefield@1wh.nhs.uk

O. Wadoo

John Moores University, Liverpool, UK

e-mail: Owadoo@nhs.net

A. Sajjad

North East Lincolnshire Care Trust Plus, Grimsby, UK

e-mail: Aamer.sajjad@nhs.net
Stage two determines the veracity of data on a subgroup of 73 pregnant women referred for previous postpartum depression (PPD), $58 \%$ of whom disclosed an episode of self-harm with the 'intent to kill themselves' to the maternity staff when they first booked in for antenatal care. Previous PPD accounted for the largest majority of referrals ( $32 \%$ ) to the PMHT followed by depression (27\%) and self-harm (10\%). The majority of women $(85 \%)$ referred to the PMHT were engaged. Eight percent were so unwell at the point of referral they required an admission to the hospital. Attempted suicide in the subgroup of 73 women with previous PPD ranged from $24-49 \%$. The findings from this audit suggest that self-harm in PPD warrants further investigation.

Keywords Self-harm $\cdot$ Postpartum depression $\cdot$ Antenatal care

\section{Introduction}

Mental disorder following childbirth is common. Around 10 to $15 \%$ of newly delivered women will experience a major depressive episode, 3 to $5 \%$ of which will be moderate to severe depression (Oates 2000). Two per 1,000 women will be admitted to the hospital for postpartum psychosis and 2 per 1,000 for a non-psychotic disorder (Oates 2000). An episode of mental illness following a subsequent delivery occurs in $50 \%$ of women with a history of severe mood disorder and $70 \%$ of women with a further family history of postpartum psychosis (Jones and Craddock 2001). Suicide is known to be a leading cause of death in the first 12 months postpartum in the UK (ConfidentialEnquiry into Maternal and Child Health 2004, 2007). In the most recent Confidential Enquiry (2011), $59 \%$ of maternal suicides were due to psychosis or severe 
depression. Most of the women ( $76 \%$ ) were married or living in stable cohabitation. More than three quarters were either employed or housewives with employed partners. As in previous enquiries, the majority of women ( $87 \%$ ) died violently from hanging, jumping from height or self-immolation (Oates and Cantwell 2011). However, little is known regarding selfharm in the postpartum period despite suicide being a leading cause of death and high rates of morbidity during this time. There have only been four studies examining this issue, two conducted here in the UK and two in the USA using the data from the same study.

The first was a consecutive 6-month sample of women age 15-44 years attending a local emergency department for non-fatal self-harm in a single catchment area in London (Appleby and Turnbull 1995). The only information collected was on the method of self harm and any deliveries in the previous 12 months. The rate of self-harm (odds ratio 0.43 ) in the postpartum period was estimated to be about half the rate for non-postpartum women of the same age. The main method of self-harm in this study was self-poisoning. The authors concluded that the rate of self-harm the year following delivery is low despite high rates of mental disorder. As part of a national audit of admissions to mother and baby units, Salmon et al. (2004) examined predictors of maternal self-harm. The only independent predictor was depressed mood, with mothers who had harmed themselves at least four times more likely to experience depressed mood during admission than those who had not.

Schiff and Grossman (2006) compared 520 women hospitalised for a suicide attempt in the postpartum period with 2,000 controls to see if there was any association between maternal complications and suicide attempt. They found that maternal complications were not associated with suicide attempt, but that fetal death or death of the infant in the first year was strongly associated. Those who attempted suicide tended to be younger, from black and minority ethnic groups and socially deprived. The main method of self-harm was selfpoisoning. The rate of attempted suicide in the postpartum period was estimated to be approximately two thirds the rate for non-postpartum women of the same age. Comtois et al. (2008) used the data from the same study to examine preexisting psychiatric risk factors for suicide attempt. They found that a prior mental health, substance use or dual diagnosis all significantly increased the risk of a suicide attempt in the postpartum period.

Many acts of self-harm do not come to the attention of healthcare services, so it is possible that the rates quoted in the studies above are underestimates as they are based on hospital attendances only (Hawton et al. 2001; Meltzer et al. 2002). An individual episode of self-harm might be an attempt to end life, but some acts of self-harm are not directly connected to suicidal intent (NICE 2004). As the term 'self-harm' is a broad one, The World Health Organisation defines it as 'an act with non-fatal outcome, in which an individual deliberately initiates a non-habitual behaviour that, without intervention from others, will cause self-harm, or deliberately ingests a substance in excess of the prescribed or generally recognised therapeutic dosage, and which is aimed at realising changes which the subject desired via the actual or expected physical consequences' (Platt et al. 1992; NICE 2004). Self-harm is more common in young women, people who are disadvantaged in socio-economic terms and those who are single or divorced, live alone, are single parents or have severe lack of social support (Meltzer et al. 2002). Following an act of selfharm, the rate of suicide increases between 50 and 100 times the rate of suicide in the general population (Hawton et al. 2003; Owens et al. 2002). Women who self-harm have an increased risk of suicide which is likely to be further enhanced by the presence of depression during pregnancy and in the postpartum period. Self-harm during pregnancy is estimated to be about half the rate for non-pregnant women (Greenblatt et al. 1997; Weiss 1999). Previous loss of a child, potential loss of a lover and the desire to have a termination were found to be associated with attempted suicide during pregnancy in one study (Lester and Beck 1988). In a further four studies, those suffering domestic violence, dysfunctional family relationships, antenatal depression and socio-economic deprivation were most at risk for attempted suicide during pregnancy (Bayatpour et al. 1992; Farber et al. 1996; Marishane and Moodley 2005; Asad et al. 2010).

As suicide is a leading cause of maternal death in England, the National Institute of Clinical Excellence (NICE 2007) and the Confidential Enquiries (Oates and Cantwell 2011) recommended that all mental health trusts in England have specialised perinatal mental health teams to care for pregnant and postpartum women. They proposed that all pregnant women should be asked about personal mental health history and family history when they first book in for antenatal care. Women with a previous history of serious affective (depression and bipolar disorder) or other psychoses should be referred in pregnancy for psychiatric assessment and management even if they are well. A minimum requirement for management should be regular monitoring and support for at least 3 months following delivery (Oates and Cantwell 2011). There is however a lack of perinatal mental health service provision in the UK with less than a quarter of NHS trusts having both inpatient and outpatient community services (Oluwatayo and Friedman 2005; Rowan and Bick 2008; Green et al. 2008). Consequently, there have only been a handful of studies describing the clinical and socio-demographic characteristics of pregnant women attending perinatal services in the UK and abroad, particularly women who self-harm (Green et al. 2008; Rowen et al. 2010; Wilson et al. 2004).

The aim of this study was to describe the clinical and socio-demographic characteristics of pregnant women referred to a new perinatal mental health team (PMHT) with 
an estimated date of delivery from April 2009 to March 2010. In accordance with the National Institute of Clinical Excellence (NICE 2007) and the Clinical Negligence Scheme for NHS Trusts guidelines (Litigation Authority 2010), data on care pathways would also be collected including the information on those who were referred but refused to engage with the PMHT. The aim of the second stage of the study was to determine the veracity of data recorded for a subgroup of 73 pregnant women referred to the team for previous postpartum depression (PPD), $58 \%$ of whom disclosed an episode of self-harm with the 'intent to kill themselves' to the maternity staff when they first booked in for antenatal care.

\section{Methods}

The perinatal mental health team

The PMHT is based in the antenatal unit at Liverpool Women's Hospital, the largest single site maternity hospital in Europe delivering around 8,000 babies a year. The team consists of one consultant psychiatrist, one consultant obstetrician, a specialist disability midwife, two psychiatric nurses and at times, a senior psychiatric trainee. At the time of the audit, the clinic was run 1 day/week at Liverpool Women's Hospital, and from January 2010, twice each month at a different site, the University Hospital Aintree. Those attending antenatal care at the University Hospital Aintree were booked in for delivery at Liverpool Women's Hospital. The PMHT was only resourced to offer care to pregnant women up to the point of delivery, despite NICE (2007) and the Confidential Enquiry team recommendations (Confidential Enquiry into Maternal and Child Health 2004, 2007, 2011).

The referral process

The majority of referrals come from midwives who screen for past or present serious mental disorder, including a history of deliberate self-harm during the booking interview. The focus of the questions is based on the NICE Guideline on Antenatal and Postnatal Mental Health (NICE 2007). The team also accepts referrals from mental health teams, family doctors (general practitioners) and obstetricians. All women booking in for antenatal care are referred to the PMHT if they meet the criteria for a significant history. The criteria for a significant mental health history are determined using the screening algorithm (Fig. 1).

Methods: stage one

An audit tool was designed to collect clinical and sociodemographic data in three sections from Liverpool Women's Hospital. Section one recorded information on the reason for
Fig. 1 Screening algorithm for significant mental health history

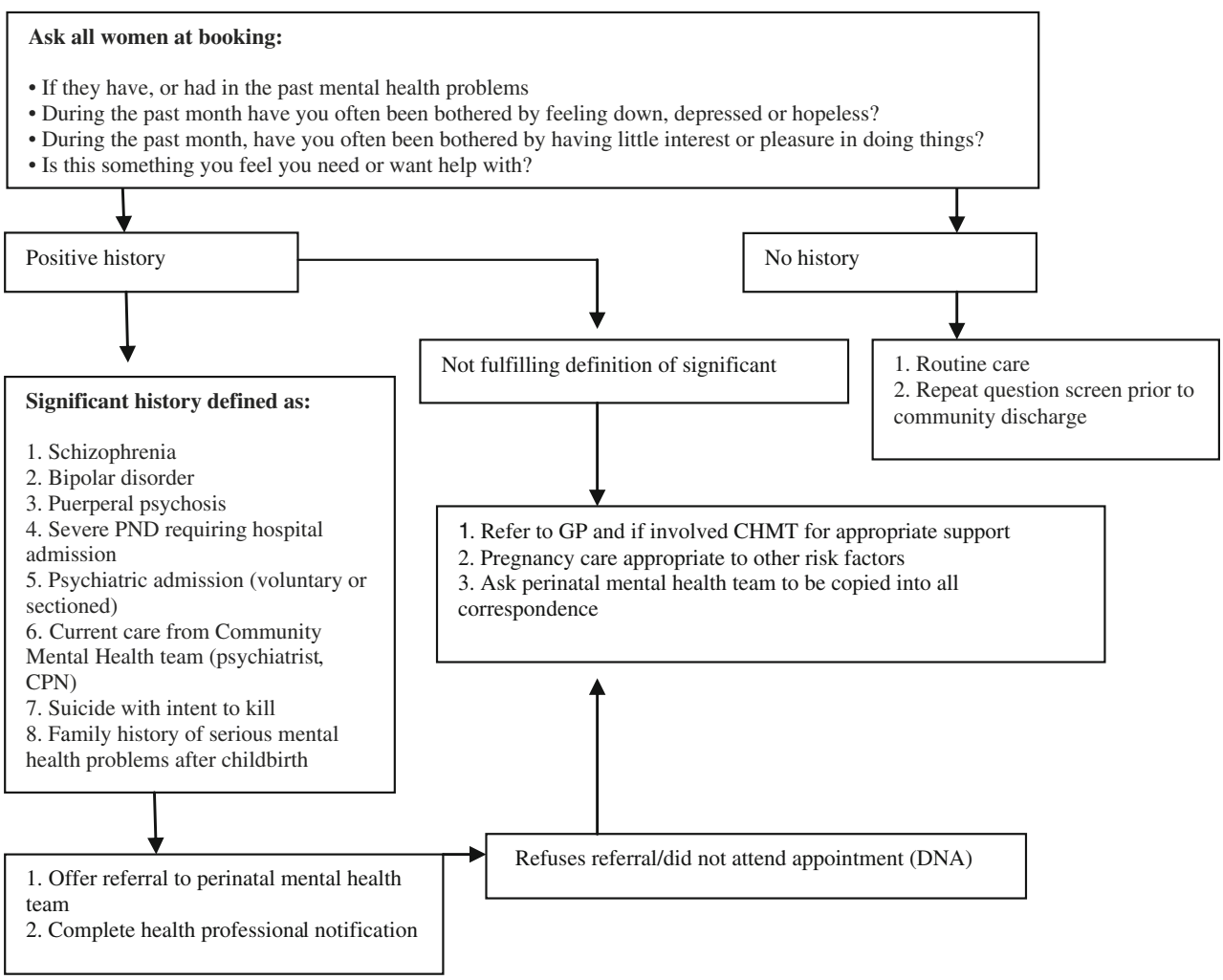


referral sent to the PMHT on standard hospital referral forms. Section two collected data from the PMHT paper case notes on the following: current and/or past mental health diagnoses, current medications, psychiatric admissions during this pregnancy, child protection/safeguarding issues and outcome of referral. Child protection/safeguarding issues were defined as a documented care or supervision order recorded in the PMHT case notes or a documented referral made to the safeguarding lead due to professional concerns. Section three recorded socio-demographic data extracted from electronic patient records at Liverpool Women's Hospital on the following: age, marital status, postcode, gravida, parity, smoking and alcohol use. Additional mental health data recorded by the maternity staff at the first antenatal booking in appointment was also collected from electronic patient records. This information was recorded in the following yes/no format: (1) significant mental health issues in the past or present, (2) previous psychiatric admissions, (3) presently in contact with a psychiatrist/community mental health team, (4) history of suicide attempts/self-harm and (5) close family history of mental health issues after childbirth. Data on domestic violence were not available to the audit team as these were dealt with by a separate specialist team. Postcodes were used to derive Index of Multiple Deprivation scores (Department for Communities and Local Government 2010). The English index of deprivation (IMD) is a measure of multiple deprivations. The model is based on the idea of distinct dimensions of deprivation which can be recognised and measured separately. These are experienced by people living in an area. These domains include employment deprivation, health deprivation and disability, education skills and training deprivation, barriers to housing and services, living environment deprivation and crime. The IMD comprises five deprivation quintiles with quintile one the least deprived and quintile five the most deprived $20 \%$ of the UK population.

\section{Procedure: stage one}

The audit was conducted by a research associate $(\mathrm{CH})$ and two senior psychiatry trainees (OW and AS). The psychiatry trainees were responsible for collecting data on mental health history in terms of reason for referral to the PMHT, current and/or past mental health diagnoses, current medications, psychiatric admissions during the current pregnancy and child protection/safeguarding issues. If there was any uncertainty regarding a mental health diagnosis or a referral was made to the PMHT for more than one diagnosis, the two psychiatry trainees used their clinical expertise and agreed on a primary diagnosis based on all the information available. $\mathrm{CH}$ was responsible for collecting socio-demographic and additional mental health data from electronic patient records at Liverpool Women's Hospital.
Methods: stage two

Stage two set out to determine the veracity of data recorded for a subgroup of 73 pregnant women referred for previous PPD. Forty-two $(58 \%)$ had an episode of self-harm recorded on electronic patient records. According to the screening algorithm provided to the maternity staff, 'suicide attempt' should only be ticked positive if the woman has declared a history of self-harm with the intent to kill herself. However, due to the way information is recorded in a simple yes or no format (with no further information available), it was possible that a suicide attempt may have taken place but that it was outside of any episode of PPD, such as a one-off impulsive overdose that was not related to childbirth. It was also possible that self-harm without suicidal intent may have been ticked positive in cases where there was no suicidal intent, such as experiencing suicidal ideation but not acting upon it or for an accidental overdose. Previous postnatal depression at the severity laid out in the screening algorithm may have also been misdiagnosed or mislabelled by midwives and obstetricians. A simple data collection tool was designed to ascertain if there was (1) any evidence of self-harm with or without suicidal intent prior to any delivery, (2) evidence of PPD defined as a documented diagnosis, a documented deterioration in symptoms of depression and a documented change in treatment or intervention and (3) any evidence of self-harm with or without suicidal intent in the postpartum period. For each case, the team would make a clinical decision as to whether a suicide attempt in PPD was definite, possible, unknown or not present.

Procedure: stage two

Access was requested to view the medical records at Liverpool Women's Hospital, Mersey Care NHS Trust (mental health care provider) and primary care (family doctor). The data from Liverpool Women's Hospital and GP case notes would be collected by $\mathrm{CH}$. The data from Mersey Care NHS Trust would be collected by the two psychiatry trainees.

Ethical approval

\section{Stage one}

Approval was granted to undertake the audit from Liverpool Women's Hospital NHS Foundation Trust Auditing Department.

\section{Stage two}

An application was made to the NHS research ethics committee to seek permission to view the medical records in order to determine whether suicide attempt in previous PPD was definite, possible, unknown or not present. As all the 
women delivered their babies between March 2009 and March 2010, it was unlikely that they would still be in contact with the maternity hospital, and thus, they are difficult to contact. The local research ethics committee recommended written informed consent in order to view the medical records. A letter and reply slip approved by the ethics was sent out on two occasions with a stamp addressed envelope. The research team did not receive a single response.

\section{National information governance board approval}

An application was then made to The National Information Governance Board for Health and Social Care (NIGB) for section 251 approval. This allows researchers in situations where consent cannot be obtained to access patient data if it is deemed to be in the public interest. The NIGB granted permission for a member of the PMHT to contact general practitioners (family doctors) to request information on the timing of any previous episode(s) of self-harm in relation to the timing of any previous episodes of PPD. No further information could be requested in terms of the actual method of self-harm or its potential lethality.

\section{Analysis}

Data were entered anonymously into SPSS version 18 by $\mathrm{CH}$ and subject to summary statistics. Descriptive statistics were used to describe the clinical and socio-demographic characteristics of women referred to the PMHT. Due to the small numbers in some diagnostic groups, no formal hypothesis tests were undertaken.

\section{Results}

Demographic characteristics

Between March 2009 and March 2010, 225 pregnant women were referred to the perinatal mental health team for a significant mental health history. This translates to a referral rate of $2.81 \%$ of 8,000 deliveries a year. Table 1 presents the demographic information on those referred.

Primary diagnosis at the point of referral

Those with PPD accounted for the largest percentage of referrals $(32 \%)$ followed by depression $(27 \%)$ and selfharm (10\%). Fifty-five percent were referred for more than one mental health diagnosis including depression, substance use disorder, anxiety, self-harm, obsessive-compulsive disorder and eating disorder. Ten percent were referred for current symptomatology, mostly depression. The primary diagnosis at the point of referral is illustrated in Table 2.
Table 1 Demographic data for 225 women referred to the PMHT

\begin{tabular}{ll}
\hline & Value \\
\hline Age & \\
Mean age in years (SD) & $29(6.2)$ \\
Marital status (number (\%)) & \\
Single & $164(73)$ \\
Married/cohabiting & $47(21)$ \\
Divorced/separated & $3(1.3)$ \\
Widowed & $2(1)$ \\
Ethnicity (number (\%)) & \\
White & $199(88)$ \\
Black & $3(1)$ \\
Black African & $7(3)$ \\
Indian/Pakistani/Bangladeshi & $2(1)$ \\
Chinese & $1(0.5)$ \\
White (other) & $4(2)$ \\
Mixed & $9(4)$ \\
Occupation ${ }^{\text {a }}$ (number (\%)) & \\
Professional/managerial & $13(6)$ \\
Skilled non-manual & $12(5)$ \\
Partly skilled & $29(30)$ \\
Unskilled & $4(2)$ \\
Unemployed & $73(32)$ \\
Mother/housewife & $13(6)$ \\
IMD score (number (\%)) & $9(4)$ \\
Score $\geq 35$ (quintile 5) & $167(75)$ \\
&
\end{tabular}

${ }^{\mathrm{a}}$ Does not add up to $100 \%$ due to missing data

Table 2 Primary diagnosis at the point of referral

\begin{tabular}{ll}
\hline Diagnosis & Number (\%) \\
\hline Previous postpartum depression & $73(32)$ \\
Depression & $61(27)$ \\
Self-harm & $22(10)$ \\
Anxiety disorder & $18(8)$ \\
Personality disorder & $12(5)$ \\
Bipolar disorder & $10(4)$ \\
Schizophrenia & $7(3)$ \\
Substance use disorder & $5(2)$ \\
Eating disorder & $5(2)$ \\
Schizoaffective disorder & $4(2)$ \\
One-off transient psychotic episode & $2(1)$ \\
Obsessive-compulsive disorder & $2(1)$ \\
Bereavement & $2(1)$ \\
Previous postpartum psychosis & $1(0.5)$ \\
Post-traumatic stress disorder & $1(0.5)$ \\
Total & $225(100)$ \\
\hline
\end{tabular}




\section{Clinical data}

Table 3 presents the data on current medications, admissions during this pregnancy, child protection issues, previous psychiatric admissions, current care, family history and self-harm.

Forty percent were not taking psychotropic medication, $75 \%$ were not under the care of a psychiatrist or community mental health team and $52 \%$ had self-harmed in the past. Twenty-six percent had experienced previous psychiatric admissions, $20 \%$ had a family history of serious mental health problems following childbirth and approximately a fifth had child protection issues.

Outcome of referral to the perinatal mental health team

In accordance with the National Institute of Clinical Excellence (NICE 2007) and the Clinical Negligence Scheme for NHS Trusts guidelines (Litigation Authority 2010), data on the outcome of every referral (up to the point of delivery) were collected including the information on those who were referred but refused to engage with the PMHT. Of the 225 referrals, 82 women $(36 \%)$ were followed up by the PMHT to the point of delivery. Fifteen percent did not attend (DNA) their appointment, and $40 \%$ were discharged after an initial assessment by the PMHT to primary care or secondary mental health services. Seventeen women ( $8 \%$ ) were unwell enough to require a psychiatric admission. Two thirds of those admitted had self-harmed in the past, and $50 \%$ had experienced previous psychiatric admissions. Those with a diagnosis of depression (34\%) and previous PPD (30\%) accounted for two thirds of admissions.

Suicide attempt in previous postpartum depression

Stage two of this study set out to determine the accuracy of data recorded for $42(58 \%)$ pregnant women referred to the PMHT for previous PPD and self-harm. Nineteen cases were verified from the information recorded by hand in the

Table 3 Mental health history for 225 women referred to the PMHT

\begin{tabular}{ll}
\hline & $\begin{array}{l}\text { Number } \\
(\%)\end{array}$ \\
\hline Currently taking medication & $135(60)$ \\
Admissions to hospital during this pregnancy & $17(8)$ \\
Safeguarding/child protection issues & $47(21)$ \\
Previous psychiatric admission(s) & $58(26)$ \\
Currently under the care of a psychiatrist/CMHT & $57(25)$ \\
$\begin{array}{l}\text { Family history of serious mental health } \\
\text { problems following childbirth }\end{array}$ & $44(20)$ \\
$\begin{array}{l}\text { Previous episode(s) of self-harm disclosed to maternity } \\
\text { staff at antenatal booking in interview }\end{array}$ & $117(52)$ \\
\hline
\end{tabular}

PMHT case notes. There were 16 cases of 'definite' suicide attempt in the PPD. The method of self-harm in all these cases was self-poisoning. There were three cases of selfharm taking place outside of an episode of PPD. Letters were sent to the general practitioners (family doctor) of the remaining 23 women to confirm or refute an episode of selfharm in PPD. This is illustrated in the flow chart (Fig. 2).

Seventeen of 23 GPs (74\%) responded to the request for additional information on the timing of previous self-harm in relation to previous PPD. The responses are summarised in Table 4.

At the end of stage two, 73 pregnant women referred to the PMHT for previous PPD $(n=73)$ fell into one of the three groups: (1) Group one accounted for $24 \%$ and were those with a definite suicide attempt in PPD. (2) Group two accounted for $25 \%$ and were those with a 'possible' suicide attempt in PPD. The GPs confirmed a history of repeat selfharm in eight (44\%) cases, but the level of suicidal intent and timing of episode(s) in relation to previous PPD was unknown. (3) Group three accounted for $52 \%$ and were those (a) referred to the PMHT for PPD only and (b) those whose self-harm was not related to PPD and four cases refuted by GPs. Table 5 presents the socio-demographic and clinical characteristics for the three groups.

Attempted suicide in this group of women referred to the PMHT for previous PPD could range from $24 \%$ to as high as $49 \%$. Six out of 35 women (groups one and two) with a history of self-harm and PPD were self-harming during the current pregnancy compared to 1 in 38 for women with previous PPD only (group three). Four out of 35 women with a history of self-harm and PPD required a psychiatric

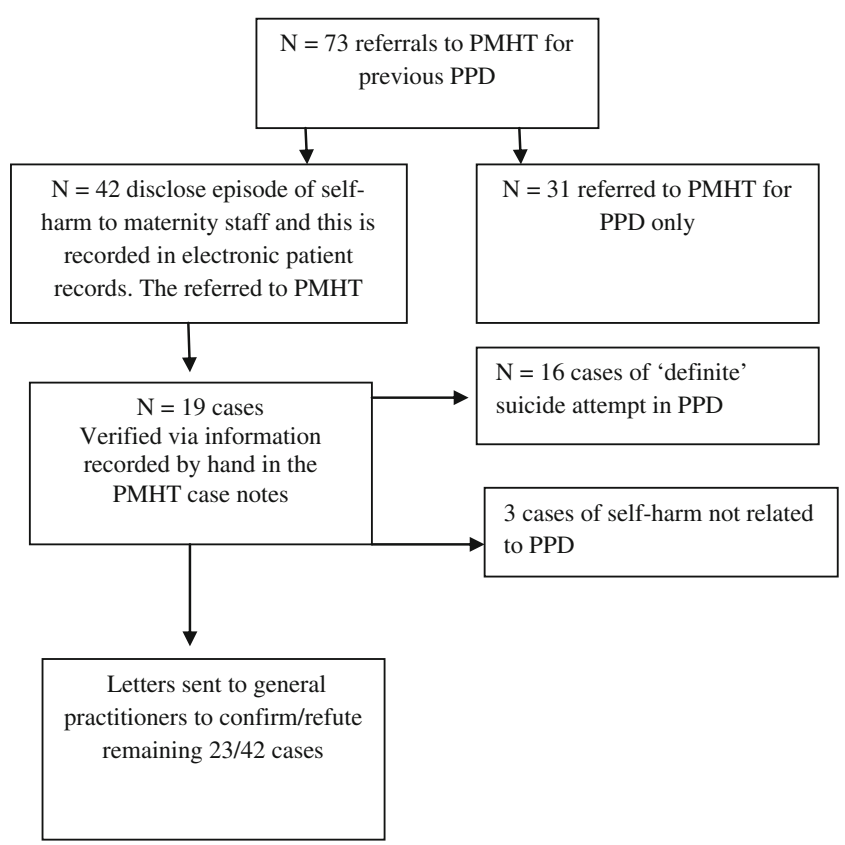

Fig. 2 Self-harm/suicide attempt in previous PPD 
Table 4 Family doctor responses

\begin{tabular}{ll}
\hline Response & Number (\%) \\
\hline $\begin{array}{l}\text { History of repeat self-harm recorded but not } \\
\text { able to confirm or refute if any episode(s) } \\
\text { took place in the postpartum period }\end{array}$ & $8(47)$ \\
$\begin{array}{l}\text { Patient no longer registered with practice } \\
\text { No episode(s) of self-harm recorded }\end{array}$ & $4(23.5)$ \\
$\begin{array}{l}\text { Confirmed an episode of self-harm } \\
\text { in the postpartum/PPD }\end{array}$ & $4(23.5)$ \\
\end{tabular}

admission during the current pregnancy compared to 1 in 38 for women with previous PPD only. A greater percentage of women in group one (47 \%) was currently suffering from depression compared to women in group two (33\%) and group three (32\%), yet only $5 \%$ were currently under the care of a psychiatrist despite a previous suicide attempt. In group two, the possible cases seemed to differ from the other two groups in that a larger number of women in this group had child protection issues, previous psychiatric admissions and be currently under the care of a psychiatrist. All groups had an Index of Multiple Deprivation score of 35 or above indicating extremely high levels of social deprivation across all three groups.

\section{Discussion}

The findings from this audit suggest that screening for a significant mental health history at the first antenatal appointment identifies those at risk of psychiatric complications during pregnancy. Over $50 \%$ of those referred to the
PMHT had self-harmed in the past, $8 \%$ were unwell enough to require a psychiatric admission and $21 \%$ had child protection issues. A referral rate of $2.81 \%$ is efficient as clearly these women did have significant current as well as past mental health issues. At the time of the audit, the PMHT was only resourced to offer care to pregnant women up to the point of delivery, despite NICE (2007) recommendations. However, $75 \%$ of those referred to the PMHT and around half of those with the most severe mental disorders were not under the care of a psychiatrist or specialist mental health team. Had the risks in the postpartum period not been identified by the PMHT, these women may have easily slipped through the net. The pregnant woman who failed to attend appointments had a health professional notification completed by the PMHT to inform the family doctor and if involved secondary mental health services of the pregnancy. Interestingly, a high percentage ( $85 \%$ ) of the total number referred did engage with the PMHT, and the risks during pregnancy were managed up to the point of delivery. Forty percent of referrals were referred on to primary or secondary mental health services after an initial assessment by the PMHT. The reason for this was that the women were currently doing well and considered not to be at high risk of recurrence after delivery. Again, the GP and if involved secondary mental health services were informed of the pregnancy, and the PMHT copied into all future correspondence up to the point of delivery. Similar to the findings of Green et al. (2008), those with psychotic disorders including schizophrenia, bipolar disorder and schizoaffective disorder accounted for only $9 \%$ of the total number of referrals, despite referrals being accepted from family doctors, psychiatrists and secondary mental health services. Referrals for

Table 5 Socio-demographic and clinical characteristics for those referred for PPD

\begin{tabular}{|c|c|c|c|}
\hline & $\begin{array}{l}\text { Definite suicide attempt } \\
\text { in PPD } n=17(24 \%)\end{array}$ & $\begin{array}{l}\text { Possible suicide attempt } \\
\text { in PPD } n=18(25 \%)\end{array}$ & $\begin{array}{l}\text { PPD only } \\
\mathrm{n}=38(52 \%)\end{array}$ \\
\hline Mean age in years $(\mathrm{SD})$ & $28(5.6)$ & $28(7.11)$ & $30(5.7)$ \\
\hline Married & $2(12 \%)$ & $2(11 \%)$ & $12(34 \%)$ \\
\hline Ethnicity/white British & $17(100 \%)$ & $15(83 \%)$ & $37(97 \%)$ \\
\hline Currently taking psychotropic medication & $9(53 \%)$ & $8(44 \%)$ & $25(65 \%)$ \\
\hline Child protection/safeguarding issues & $1(5 \%)$ & $6(33 \%)$ & $6(16 \%)$ \\
\hline Psychiatric admissions during this pregnancy & $1(5 \%)$ & $3(16 \%)$ & $1(3 \%)$ \\
\hline Previous psychiatric admission(s) & $2(12 \%)$ & $9(50 \%)$ & $5(14 \%)$ \\
\hline Currently under the care of community mental health team/psychiatrist & $1(5 \%)$ & $8(44 \%)$ & $7(19 \%)$ \\
\hline Current self-harm during this pregnancy & $2(12 \%)$ & $4(22 \%)$ & $1(3 \%)$ \\
\hline Current depression during this pregnancy & $8(47 \%)$ & $6(33 \%)$ & $12(32 \%)$ \\
\hline Family history of mental health issues following childbirth & $1(5 \%)$ & $4(22 \%)$ & $9(24 \%)$ \\
\hline IMD score 35 or more (quintile 5 ) & $14(87 \%)$ & $15(83 \%)$ & $29(78 \%)$ \\
\hline Any obstetric complication recorded with delivery resulting in PPD & $7(41 \%)$ & $3(16 \%)$ & $6(17 \%)$ \\
\hline PPD recorded with more than one previous delivery & $1(5 \%)$ & $4(22 \%)$ & $7(18 \%)$ \\
\hline
\end{tabular}

Total $n=73$ 
these disorders have gradually increased since the audit took place, but the reason for the low numbers is unknown. One possible explanation is that family doctors and mental health staff may not realise that bipolar disorder and psychosis are risk factors for puerperal psychosis. It is possible to project the number of postpartum women, including those with psychotic disorders, likely to need specialist perinatal mental health care. (National Institute for Clinical Excellence 2008) recommends using 40 per 1,000 deliveries in estimating a benchmark for referral to a specialist perinatal mental health team. For 8,000 deliveries, this translates to 320 women per year, including the 4 per 1,000 deliveries to women who are likely to require admission to hospital (National Institute for Clinical Excellence 2008).

During stage one of the audit, the extraordinarily high rate of attempted suicide in previous PPD became apparent, and the final figure could be as high as $49 \%$ in this subgroup. As self-harm was a reason to warrant referral to the PMHT, a high rate in those attending might be expected, but a diagnosis of severe mental disorder, severe PPD requiring hospital admission, current care from a psychiatrist, current care from a mental health team and family history were also reasons for referral. Those referred for past or current self-harm and no other mental health diagnoses made up only $10 \%$ of the total number of referrals with those referred for previous PPD accounting for the majority of cases of past self-harm. This suggests that self-harm in PPD is an issue that warrants further investigation. Only $9 \%$ of those with definite and possible suicide attempt were in touch with secondary mental health services, despite $40 \%$ presenting with current symptomatology. A greater number of women with a history of previous self-harm and PDD were self-harming during the current pregnancy and admitted to the hospital, which suggest that self-harm in previous PPD might be a risk factor for self-harm in subsequent pregnancies. It is important to note that even if self-harm takes place outside of an episode of PPD, the risk for suicide is still increased, a risk likely to be enhanced by a relapse of depression during pregnancy and the postpartum period. The possible cases of attempted suicide, including the $44 \%$ who repeatedly self-harmed, shared many of the characteristics known to be associated with women who self-harm. Similar to the findings of Schiff and Grossman (2006), they were mostly young, single, experienced high levels of social deprivation and were known to child protection services. This is in contrast to the suicides reported to the Confidential Enquiries. These women tended to be older, married or in stable cohabitation and employed (Oates and Cantwell 2011). But, the team were unable to confirm or refute a definite suicide attempt in these cases due to not being able to access additional medical records. This was also the case in determining a definite or probable diagnosis of previous PPD. The higher proportion of child protection issues, current self-harm, previous and current psychiatric admissions and current care from a psychiatrist in this particular group could possibly be explained by a different diagnosis, such as borderline personality disorder (BPD). It is estimated that around 41 to $83 \%$ of those with BPD report a history of major depression (Lieb et al. 2004).

From the literature on self-harm, it is known that the method and 'reason' for self-harm can and often will change not only between episodes but also within the same episode. It is therefore very difficult to estimate the degree of suicidal intent unless it is disclosed by the individual (Williams 2001). Similar to the findings of Appleby and Turnbull (1995) and Schiff and Grossman (2006), the main method of self-harm in this study was self-poisoning. This differs to the methods of suicide in the Confidential Enquiries where the majority of women $(87 \%)$ died violently (Oates and Cantwell 2011). As this was an observational study, the odds ratio cannot be compared to the studies presented in the 'Introduction' (Appleby and Turnbull 1995; Schiff and Grossman 2006); however, it is important to note that $58 \%$ of pregnant women with previous PPD disclosed a past suicide attempt to maternity staff during the first antenatal appointment, despite the consequences of disclosing such sensitive information in terms of the child protection issues it raises.

\section{Limitations}

Due to the team being unable to access medical records without written informed consent, only 17 cases of definite attempted suicide in previous PPD were confirmed. Although the family doctors confirmed a history of repeat self-harm in $44 \%$ of the possible cases, the method of self-harm and its timing in relation to PPD remain unknown. This was also the case for a definite or possible past diagnosis of PPD at the severity laid out in the screening algorithm. Due to the small numbers in some of the groups, it was not possible to examine associations between variables or predictors for self-harm. This was also the case for those referred for previous PPD due to the uncertainty regarding the possible cases of selfharm in PPD. The team was also unable to collect data on domestic violence and follow-up data on any adverse outcomes for the mother and infant at 3 months postpartum due to not being able to gain access to medical records. A study that consents pregnant women with previous PPD at the point of entry into maternity services, with a minimum of 12 months postpartum follow-up, is needed in order to examine the relationship between self-harm and PPD and outcomes for mother and baby. The preliminary findings from this audit would suggest that self-harm in PPD is an issue that warrants further investigation since it may be a risk factor or indicator of pregnant women vulnerable to self-harm in subsequent pregnancies and the postpartum period. 
Acknowledgments National Institute for Health Research (NIHR) Flexibility and Sustainability Funding was provided by Liverpool Primary Care Trust to undertake stage two of the audit. We specially thank Professor James Neilson, Liverpool Women's Hospital for the use of the research fellows' room.

Open Access This article is distributed under the terms of the Creative Commons Attribution License which permits any use, distribution, and reproduction in any medium, provided the original author(s) and the source are credited.

\section{References}

Appleby L, Turnbull G (1995) Parasuicide in the first postnatal year. Psychol Med 25:1087-1090

Asad N, Karmaliani R, Sullaiman N, Bann C, McClure EM, Pasha O, Wright LL, Goldenberg RL (2010) Prevalence of suicidal thoughts and attempts among Pakistani women. Acta Obstet Scan 89:1545-1551

Bayatpour M, Wells R, Holford S (1992) Physical and sexual abuse as predictors of substance use and suicide among pregnant teenagers. J Adolesc Heal 13:128-132

Centre for Maternal and Child Enquiries (CMACE) (2011) Saving mothers lives: reviewing maternal deaths to make motherhood safer: 2006-2008. The eighth report on confidential enquiries into maternal deaths in the United Kingdom

Comtois KA, Schiff MA, Grossman DC (2008) Psychiatric risk factors associated with postpartum suicide attempt in Washington State, 1992-2001. American Journal of Obstetrics and Gynaecology 199:120-125

ConfidentialEnquiry into Maternal and Child Health (2004) Why mothers die 2000-2002: report of the confidential enquiries into maternal deaths in the UK. RCOG, London

Confidential Enquiry into Maternal and Child Health (2007) Saving mothers' lives: reviewing maternal deaths to make motherhood safer-2003-2005. RCOG, London

Department for Communities and Local Government (2010) English indices of deprivation: guidance document. Available from http://www.communities.gov.uk/corporate/researchandstatistics/ statistics/subject/indicesdeprivation

Farber E, Herbert S, Reviere S (1996) Childhood abuse and suicidality in obstetric patients in a hospital-based urban prenatal clinic. General Hospital Psychiatry 18:56-60

Green L, Elliot S, Anwar L, Best E, Tero M, Sarkar A, Ross-Davie M, Hodgkiss A, Hunter M, Boocock A (2008) An audit of pregnant women with severe mental illness referred during the first two years of a new perinatal mental health service. Archives of Women's Mental Health 11:149-158

Greenblatt J, Dannenberg A, Johnson C (1997) Incidence of hospitalized injuries among pregnant women in Maryland 1979-1990. American Journal of Preventive Medicine 13:374-379

Hawton K, Harriss L, Hodder K et al (2001) The influence of the economic and social environment on deliberate self-harm and suicide: an ecological and person-based study. Psychol Med 31:827-836

Hawton K, Zahl D, Weatherall R (2003) Suicide following deliberate self-harm: long-term follow-up of patients who presented to a general hospital. Br J Psychiatry 182:537-542
Jones I, Craddock N (2001) Familiality of the puerperal trigger in bipolar disorder: results of a family study. Am J Psychiatry 158:913-917

Lester D, Beck AT (1988) Attempted suicide and pregnancy. Am J Obstet Gynecol 158:1084-1085

Lieb K, Zanarini M, Schmahl C, Linehan M, Bohus M (2004) Borderline personality disorder. Lancet 364:453-461

Marishane T, Moodley J (2005) Parasuicide in pregnancy. International Journal of Gynecology and Obstetrics 89:268-271

Meltzer H, Lader D, Corbin T et al (2002) Non-fatal suicidal behaviour among adults aged 16 to 74 in Great Britain. The Stationery Office, London

National Institute for Health and Clinical Excellence (2004) Self-harm: The short-term physical and psychological management and secondary prevention of self-harm in primary and secondary care. NICE Clinical Guideline 16. NICE, London

National Institute for Health and Clinical Excellence (2007) Antenatal and Postnatal Mental Health: Clinical Management and Service Guidance. NICE Clinical Guideline 45. NICE, London.

National Institute of Clinical Excellence (2008) Antenatal and Postnatal Mental Health Services: Commissioning Guide: Implementing NICE Guidance. NICE, London

NHS Litigation Authority (2010) Clinical Negligence Scheme for Trusts Maternity Clinical Risk Management Standards: Version 1 2010/11. Available from http://www.nhsla.com/riskmanagement/

Oates M (2000) Perinatal maternal mental health services: council report CR88. Royal College of Psychiatrists, London

Oates M, Cantwell R (2011) Deaths from psychiatric causes. Br J Obstet Gynaecol 118:1-203

Oluwatayo O, Friedman T (2005) A survey of specialist perinatal mental health services in England. Psychiatr Bull 29:177179

Owens D, Horrocks J, House A (2002) Fatal and non-fatal repetition of self-harm. Systematic review. Br J Psychiatry 181:193-199

Platt S, Bille-Brahe U, Kerkhof A et al (1992) Parasuicide in Europe: the WHO/EURO multicentre study on parasuicide. I. Introduction and preliminary analysis for 1989. Acta Psychiatr Scand 85:97104

Rowan C, Bick D (2008) An evaluation of the provision of perinatal mental health services in two English strategic health authorities. Evidence Based Midwifery 6:76-82

Rowen C, McCourt C, Bick D (2010) Provision of perinatal mental health services in two English strategic health authorities: views and perspectives of the multi-professional team. Evidence Based Midwifery 8(3):98-106

Salmon MP, Abel K, Webb R, Wharburton L, Appleby L (2004) A national audit of joint mother and baby admissions to UK psychiatric hospitals: an overview of findings. Archives of Women's Mental Health 7:65-70

Schiff M, Grossman D (2006) Adverse perinatal outcomes and risk for postpartum suicide attempt in Washington State, 1987-2001. Pediatrics 118:669-675

Weiss H (1999) Pregnancy-associated injury hospitalizations in Pennsylvania, 1995. Ann Emerg Med 34:626-636

Williams M (2001) Suicide and attempted suicide. Penguin Books Ltd, London

Wilson D, Bobier C, MacDonald E (2004) A perinatal psychiatric service audit in New Zealand: patient characteristics and outcomes. Archives of Women's Mental Health 7:71-99 